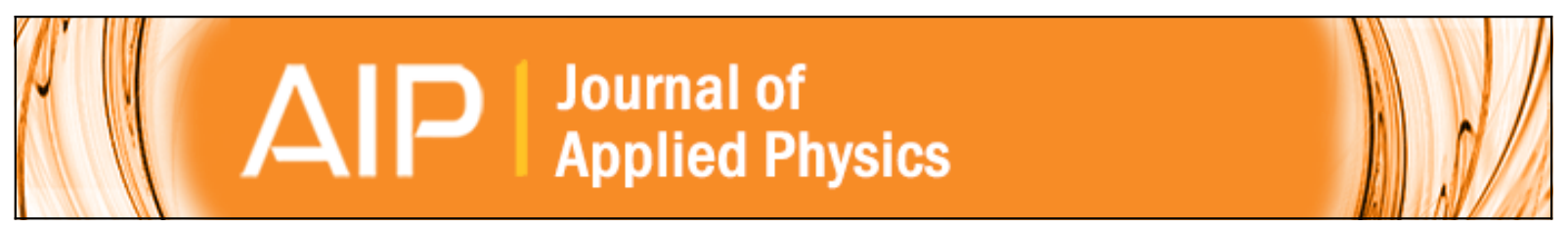

\title{
Determination of elastic properties of a MnO2 coating by surface acoustic wave velocity dispersion analysis
}

J. Sermeus, R. Sinha, K. Vanstreels, P. M. Vereecken, and C. Glorieux

Citation: Journal of Applied Physics 116, 023503 (2014); doi: 10.1063/1.4885427

View online: http://dx.doi.org/10.1063/1.4885427

View Table of Contents: http://scitation.aip.org/content/aip/journal/jap/116/2?ver=pdfcov

Published by the AIP Publishing

\section{Articles you may be interested in}

Brillouin scattering determination of the surface acoustic wave velocity in $\ln \times \mathrm{Ga} 1$ x N: A probe into the elastic constants

Appl. Phys. Lett. 101, 062103 (2012); 10.1063/1.4744961

X-ray diffraction and surface acoustic wave analysis of BST/Pt/TiO2/SiO2/Si thin films

J. Appl. Phys. 110, 104506 (2011); 10.1063/1.3662188

Determination of the high pressure elasticity of cobalt from measured interfacial acoustic wave velocities

Appl. Phys. Lett. 89, 111920 (2006); 10.1063/1.2220537

Surface acoustic wave investigations of the metal-to-insulator transition of $\mathrm{V} 2 \mathrm{O} 3$ thin films on lithium niobate J. Appl. Phys. 98, 084111 (2005); 10.1063/1.2103410

Temperature stable LiNb O 3 surface acoustic wave device with diode sputtered amorphous Te O 2 over-layer Appl. Phys. Lett. 86, 223508 (2005); 10.1063/1.1944231

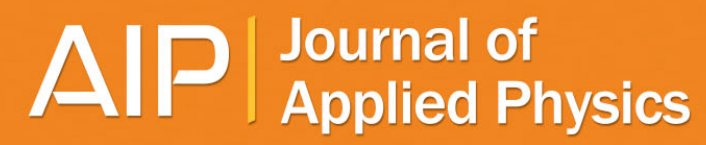

Journal of Applied Physics is pleased to announce André Anders as its new Editor-in-Chief 


\title{
Determination of elastic properties of a $\mathrm{MnO}_{2}$ coating by surface acoustic wave velocity dispersion analysis
}

\author{
J. Sermeus, ${ }^{1}$ R. Sinha, ${ }^{2,3}$ K. Vanstreels, ${ }^{2}$ P. M. Vereecken, ${ }^{2,3}$ and C. Glorieux ${ }^{1, a)}$ \\ ${ }^{1}$ Laboratory for Acoustics and Thermal Physics, KU Leuven, University of Leuven, Celestijnenlaan 200D, \\ $B$-3001 Heverlee, Belgium \\ ${ }^{2}$ IMEC, Kapeldreef 75, B-3001 Leuven, Belgium \\ ${ }^{3}$ Center for Surface Chemistry and Catalysis, KU Leuven, University of Leuven, Kasteelpark Arenberg 23, \\ B-3001 Leuven, Belgium
}

(Received 23 April 2014; accepted 14 June 2014; published online 9 July 2014)

\begin{abstract}
$\mathrm{MnO}_{2}$ is a material of interest in the development of high energy-density batteries, specifically as a coating material for internal 3D structures, thus ensuring rapid energy deployment. Its electrochemical properties have been mapped extensively, but there are, to the best of the authors' knowledge, no records of the elastic properties of thin film $\mathrm{MnO}_{2}$. Impulsive stimulated thermal scattering (ISTS), also known as the heterodyne diffraction or transient grating technique, was used to determine the Young's modulus $(E)$ and porosity $(\psi)$ of a $500 \mathrm{~nm}$ thick $\mathrm{MnO}_{2}$ coating on a $\mathrm{Si}(001)$ substrate. ISTS is an all optical method that is able to excite and detect surface acoustic waves (SAWs) on opaque samples. From the measured SAW velocity dispersion, the Young's modulus and porosity were determined to be $E=25 \pm 1 \mathrm{GPa}$ and $\psi=42 \pm 1 \%$, respectively. These values were confirmed by independent techniques and determined by a most-squares analysis of the carefully fitted SAW velocity dispersion. This study demonstrates the ability of the presented technique to determine the elastic parameters of a thin, porous film on an anisotropic substrate. (C) 2014 AIP Publishing LLC. [http://dx.doi.org/10.1063/1.4885427]
\end{abstract}

\section{INTRODUCTION}

Today, battery development is shifted to both larger and smaller sizes. In the case of the former, one can think of uses in hybrid cars, while the latter is steered by the push towards biocompatible nano-technology that can operate inside the human body. ${ }^{1}$ This requires compact and reliable energy storage. One step towards this is through the development of batteries with 3D internal structures. A micrometer-scale 3D structure coated with Manganese Oxide $\left(\mathrm{MnO}_{2}\right)$ acting as a cathode has a large surface to volume ratio, which is vital for energy deployment. $\mathrm{MnO}_{2}$ is a known cathode but has mainly been used in its powdered form. ${ }^{2}$ The electrochemical properties of $\mathrm{MnO}_{2}$ have been mapped carefully, ${ }^{3-5}$ but to the best knowledge of the authors, there are no records of the mechanical properties of a thin $\mathrm{MnO}_{2}$ coating. These properties are necessary in the development of practical batteries, and they are of particular interest to solve the adhesion problems $\mathrm{MnO}_{2}$ has when it is deposited on a platinum substrate, which is a known biocompatible material. The quality of adhesion can be probed by surface acoustic waves (SAWs) if the elastic properties are known. ${ }^{6,7}$ The aim of this paper is, therefore, to estimate a value for the Young's modulus and porosity of a $\mathrm{MnO}_{2}$ coating.

In this work, the Young's modulus $(E)$ and the porosity $(\psi)$ of a $500 \mathrm{~nm}$ thick $\mathrm{MnO}_{2}$ coating on a titanium nitride (TiN) coated $\mathrm{Si}(001)$ substrate were determined through careful analysis of the SAW behaviour.

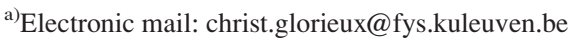

Narrowband SAWs were excited and detected by an experimental technique known as impulsive stimulated thermal scattering (ISTS), which is also known as heterodyne diffraction (HD) or transient grating (TG). In 1968, Lee and White ${ }^{8}$ were the first to excite SAW using transient surface heating by spatially periodic illumination. It was not, however, until 1981 that Nelson et al. ${ }^{9}$ introduced the ISTS method as it is known today. The specific setup that is used in this work will be described in more detail in Sec. IV. ISTS has successfully been used to study SAW behavior and to probe material characteristics for a number of different configurations, in both solid and liquid materials. Characterization of bulk solids and liquids, ${ }^{10-13}$ the study of acoustic waves at interfaces ${ }^{14,15}$ and the investigation of thin (un)supported films ${ }^{16,17}$ are just a few examples.

The SAW velocity of a coated substrate is dispersive. In the low frequency limit (or equivalently the long wavelength limit), the velocity tends towards the Rayleigh velocity for the substrate. In the high frequency (or small wavelength) limit, it tends towards the Rayleigh velocity of the coating. As the penetration depth is approximately the same as the wavelength, SAWs mainly propagate in the substrate while high frequency SAWs mainly propagate in the coating. It is possible to measure this dispersion and, through fitting, find an estimate of the elastic properties of the coating and/or the substrate. This approach has been used frequently in literature by non-contact techniques, like the one presented here, and by contact methods (see, e.g., Ref. 18). 
The used $\operatorname{Si}(001)$ substrate is a single cubic crystal and thus is anisotropic. The anisotropy of the substrate makes the SAW velocity dispersion depend both on the wavelength and on the direction of the SAW on the sample surface. In Sec. III, the theory of acoustic waves on anisotropic semi-infinite, media is briefly discussed and applied for SAW on coated substrates. Section III C discusses the simulation of the angular dispersion. The difficulties, and their solutions, of these simulations are presented. Section IV presents the experimental results of the SAW measurements, as well as the analysis of the data. The obtained elastic properties of the $\mathrm{MnO}_{2}$ coating were checked by two independent techniques, of which the results are reported in Sec. IV C. Finally, a conclusion is presented.

\section{SAMPLE PREPARATION}

In Figure 1, a cross section of the sample is shown. The sample was comprised of a $\mathrm{Si}(001)$ substrate coated by $80 \pm 10 \mathrm{~nm}$ of TiN. On top of this, a $35 \pm 5 \mathrm{~nm}$ graphite carbon layer and a $460 \pm 55 \mathrm{~nm} \mathrm{MnO}_{2}$ layer were deposited. The $\mathrm{MnO}_{2}$ coating was porous (see Figure 1) and was assumed elastically isotropic in the analysis.

The TiN layer was sputtered from a Ti target in a $\mathrm{N}_{2}$ atmosphere onto the $\mathrm{Si}(001)$ substrate. The graphitic carbon layer was grown via plasma enhanced chemical vapor deposition. ${ }^{19}$ The $\mathrm{MnO}_{2}$ film was deposited by galvanostatic electrodeposition from an aqueous solution of $0.3 \mathrm{M} \mathrm{MnSO}_{4} \times \mathrm{H}_{2} \mathrm{O}$ (Alfa Aesar) and $0.3 \mathrm{M} \mathrm{H}_{2} \mathrm{SO}_{4}$ (OM Group) at room temperature $\left(20-22^{\circ} \mathrm{C}\right)$. The current density was $0.5 \mathrm{~mA} \cdot \mathrm{cm}^{-2}$ for $600 \mathrm{~s}$, and the charge density was $300 \mathrm{mC} \cdot \mathrm{cm}^{-2}$ (targeting a theoretical thickness of $270 \mathrm{~nm}$ for a dense, non-porous film). Electrochemical deposition of the $\mathrm{MnO}_{2}$ film was executed in a three electrode setup with a Pt mesh counter electrode and a $\mathrm{Ag} / \mathrm{AgCl}(3 \mathrm{M} \mathrm{NaCl})$ reference electrode $(0.22 \mathrm{~V}$ vs. SHE). The TiN/carbon layer was used as the working electrode. After deposition, the samples were annealed at $350{ }^{\circ} \mathrm{C}$ in a $\mathrm{N}_{2}$ atmosphere (200 millibar) for $3 \mathrm{~h}$, with a $20 \mathrm{~min}$ ramp up. Next, they were allowed to cool down to room temperature.

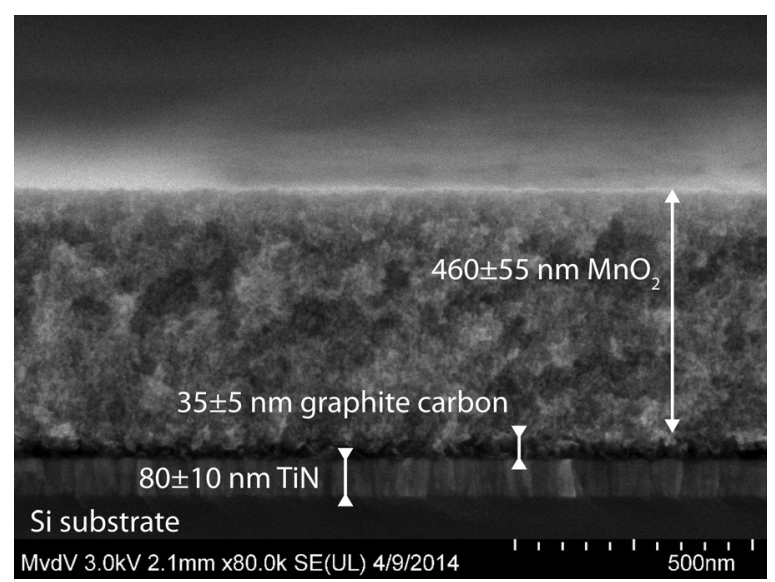

FIG. 1. SEM image of a cross-section of the sample under investigation.
The intermediate TiN layer was thin compared to the $\mathrm{MnO}_{2}$ coating but has to be accounted for in the calculation of the SAW velocity dispersion used during the fitting process. The calculated SAW velocity of a $\mathrm{Si}$ coated $\mathrm{MnO}_{2}$ system (i.e., without the TiN layer) was roughly $100 \mathrm{~m} \cdot \mathrm{s}^{-1}$ lower than the SAW velocity of the sample under investigation, based on the values of the elastic properties listed in Table I. The second intermediate layer (i.e., the graphite carbon) was combined, during the fitting procedure, with the $\mathrm{MnO}_{2}$ layer to form a $500 \pm 60 \mathrm{~nm}$ top coating, of which the elastic properties are determined. This was done because the graphite carbon is porous and, thus, will also contain some $\mathrm{MnO}_{2}$. As the elastic properties of this intermediate layer are unknown, but expected to be comparable to the ones of the top layer, it was decided to fit the data as if the sample consisted of a $500 \mathrm{~nm} \mathrm{MnO}_{2}$ top coating.

\section{THEORETICAL MODEL}

The theory for acoustic waves in anisotropic media is well understood ${ }^{22}$ and only the most relevant parts are summarized here, starting with a semi-infinite substrate. In the second part, the approach will be extended to an isotropiccoating/anisotropic-substrate system.

\section{A. General approach for semi-infinite substrate configuration}

An anisotropic substrate was modeled as a semiinfinite half-space covering the positive part of the $z$-axis and with an interface in the $x-y$ plane at $z=0$. Since the experimentally excited waves are independent of the $y$-direction and travel in both the positive and the negative $x$-directions, one can model the displacement vector $\boldsymbol{u}$ at the surface as

$$
\boldsymbol{u}=\boldsymbol{U} e^{i(k x+\xi z-\omega t)},
$$

where $k$ and $\xi$ are the wavenumbers for the $x$ and $z$ direction, respectively, and $\omega$ is the frequency. Note that, while no waves are excited in the $y$ direction, it is possible to have particle displacements in the $y$ direction.

The wave equation that follows from the combination of Newton's second law, $\rho \frac{\partial^{2} u_{i}}{\partial t^{2}}=\frac{\partial \sigma_{i j}}{\partial x_{j}}$, and Hooke's law, $\sigma_{i j}=c_{i j k l} \varepsilon_{k l}$, can be worked out, using the proposed solution in Eq. (1), as

TABLE I. Values of the physical properties of the Si substrate and TiN intermediate layer, used in the fitting procedure. For TiN $E$ values ranging from $200 \mathrm{GPa}$ to $500 \mathrm{GPa}^{21}$ can be found, here an intermediate value was used.

\begin{tabular}{lccc}
\hline \hline \multicolumn{2}{c}{ TiN } & & \multicolumn{2}{c}{$\mathrm{Si}(001)^{20}$} \\
\hline$E(\mathrm{GPa})$ & 350 & $c_{11}(\mathrm{GPa})$ & 165.35 \\
$\nu$ & 0.2 & $c_{12}(\mathrm{GPa})$ & 63.5 \\
$\rho\left(\mathrm{kg} \cdot \mathrm{m}^{-3}\right)$ & 5400 & $c_{44}(\mathrm{GPa})$ & 79.6 \\
$L(\mathrm{~nm})$ & 80 & $\rho\left(\mathrm{kg} \cdot \mathrm{m}^{-3}\right)$ & 2300 \\
\hline \hline
\end{tabular}




$$
\left(\begin{array}{lll}
k^{2} c_{11}+2 k \xi c_{15}+\xi^{2} c_{55}-\rho \omega^{2} & k^{2} c_{16}+k \xi c_{14}+k \xi c_{56}+\xi^{2} c_{54} & k^{2} c_{15}+k \xi c_{13}+k \xi c_{55}+\xi^{2} c_{53} \\
k^{2} c_{61}+k \xi c_{65}+k \xi c_{41}+\xi^{2} c_{45} & k^{2} c_{66}+2 k \xi c_{46}+\xi^{2} c_{44}-\rho \omega^{2} & k^{2} c_{65}+k \xi c_{63}+k \xi c_{45}+\xi^{2} c_{43} \\
k^{2} c_{51}+k \xi c_{55}+k \xi c_{31}+\xi^{2} c_{35} & k^{2} c_{56}+k \xi c_{54}+k \xi c_{36}+\xi^{2} c_{34} & k^{2} c_{55}+2 k \xi c_{35}+\xi^{2} c_{33}-\rho \omega^{2}
\end{array}\right)\left(\begin{array}{l}
u_{1} \\
u_{2} \\
u_{3}
\end{array}\right)=0,
$$

where $c_{I J}$ is the Voigt notation for the elements of the stiffness tensor $c_{i j k l}$ and $\rho$ is the density.

The matrix is called the Christoffel matrix, $\Gamma$, and its determinant, $\Delta \Gamma$, must be equal to zero to allow for non-trivial solutions. Given the stiffness-matrix $c_{i j k l}$, density $\rho$, frequency $\omega$, and wavenumber $k$ in the $x$-direction, the demand that $\Delta \Gamma=0$ can be used to find six wavenumbers $\xi_{j=1: 6}$ in the $z$-direction. Substituting these $\xi_{j}$ 's in Eq. (2), a vector $\boldsymbol{U}_{m, j}$, describing the null space of $\Gamma$, can be found that solves Eq. (2) for that particular $\xi_{j}$. The displacement $\boldsymbol{u}=\left(u_{x} u_{y} u_{z}\right)$ is a linear combination of the six solutions $\boldsymbol{U}_{m, j}$ in combination with their respective $\xi_{j}$

$$
u_{m=x, y, z}=\sum_{j=1}^{6} a_{j} U_{m, j} e^{i\left(k x+\xi_{j} z-\omega t\right)},
$$

with amplitudes $a_{j=1: 6}$ to be determined by boundary conditions (BCs) - see Sec. III B. From these displacements, the stresses can be calculated using Hooke's law. The stresses on the $z$-plane are given by

$$
\sigma_{k z}=\sum_{j=1}^{6} i D_{m j} a_{j} e^{i\left(k x+\xi_{j} z-\omega t\right)},
$$

with

$$
\begin{aligned}
D_{m j}= & \left(k c_{x x m z}+\xi_{j} c_{m z x z}\right) U_{x, j}+\left(k c_{m z x y}+\xi_{j} c_{y z m z}\right) U_{y, j} \\
& +\left(k c_{m z x z}+\xi_{j} c_{z z m z}\right) U_{z, j} .
\end{aligned}
$$

In the experiments, the sample was rotated in the $x-y$ plane. To account for this rotation, the stiffness matrix $c_{i j k l}$ was turned using the Bond method. ${ }^{22,23}$

\section{B. Boundary conditions in an isotropic coating- anisotropic substrate configuration}

The BCs for SAWs on a multilayered system require the continuity of displacements and stresses at the interfaces, no displacement for $z \rightarrow \infty$ (SAW are surface bound, so no displacement can exist at infinite depth), and a stress-free surface. As there are three displacement components and three stress components, a total of $6 N$ (with $N$ the number of layers) BCs can be constructed. This allows the determination of the $6 N$ unknown amplitudes $a_{j=1: 6, n=1: N}$ for all $N$ anisotropic layers. It can be shown that from the BC $u_{z}(z \rightarrow \infty)=0$, it follows that $a_{j=4: 6 \text {,substrate }}=0$, where $a_{j=4: 6, \text { substrate }}$ are the amplitudes that correspond to $\xi$ 's with a negative imaginary part. This reduces the number of unknowns to $6 N-3$.

Here, we consider an isotropic layer on an anisotropic substrate. Displacements in isotropic media are classically separated in a longitudinal and a shear component by the
Helmholtz decomposition into two potentials ${ }^{24}$ $\boldsymbol{u}=\nabla \psi+\nabla \times \phi$. It can be shown that there are, for an $N$ layered isotropic system, $4 N$ amplitudes that have to be determined by solving $4 N$ BCs. ${ }^{24}$ This means that there is a mismatch in needed BCs between the anisotropic substrate $(6 N)$ and the isotropic coating $(4 N)$. The solution to circumvent this mismatch is to treat the isotropic coating as if it were anisotropic, with the $\xi$ 's given by

$$
\begin{gathered}
\xi_{j=1: 2, c}= \pm \sqrt{k^{2}-\frac{\omega^{2}}{c_{T}^{2}},} \\
= \pm \sqrt{k^{2}-\frac{2 \rho(1+\nu) \omega^{2}}{E},} \\
\xi_{j=3: 4, c}=\xi_{j=1: 2, c}, \\
\xi_{j=5: 6, c}= \pm \sqrt{k^{2}-\frac{\omega^{2}}{c_{L}^{2}},} \\
= \pm \sqrt{k^{2}-\frac{\rho(1+\nu)(1-2 \nu) \omega^{2}}{E(1-\nu)}},
\end{gathered}
$$

where $c_{L, T}$ are the longitudinal and transversal bulk wave velocities, $E$ is the Young's modulus, $\nu$ is the Poisson's ratio, and $\rho$ is the density. Note that there are two pairs of $\xi$ 's associated with the shear wave in the isotropic coating, Eqs. (6) and (7), and only one pair with the longitudinal wave, Eq. (8).

One can write the equations representing the $\mathrm{BCs}$ as $R A=0$ with $A=\left(a_{j=1: 6, c} a_{j=1: 3, s}\right)$ and $R$ the homogenous $(6 N-3) \times(6 N-3) \mathrm{BC}$ matrix. In order to find a non-trivial solution, the determinant of $R(\Delta R)$ must be zero. The solution of $\Delta R=0$ results in a relation between $k$ and $\omega$, called the dispersion relation. It expresses the SAW velocity as a function of the frequency or equivalently as a function of the wavelength. This relation can not be described analytically, and thus, numerical simulations are needed (see Sec. III C). For the $(k, \omega)$ pairs that render a solution, the amplitudes $a_{j}$ in Eq. (3) can be found as the null-space of the BC matrix $R$.

Finally, it should be noted that in this particular situation, an anisotropic substrate is coated with two isotropic layers, i.e., the TiN intermediate layer and the $\mathrm{MnO}_{2}$ coating. All materials were treated as if they were anisotropic, resulting in a $15 \times 15 R$ matrix.

\section{Simulations of SAW velocity dispersion}

The simulated SAW velocity dispersion was used in the fitting of the experimental data to obtain the results below. In the simulation, the SAW velocity dispersion curves were calculated for a chosen wavelength $\lambda$. The simulation requires the thickness of the coating $L_{c}$ and intermediate layer $L_{i n}$, the 
densities $\rho_{s / i n / c}$, the stiffness matrix $c_{I J, s}$, the elastic constants describing the coating and intermediate layer $E_{i n / c}$ and $\nu_{i n / c}$, and the orientation of the substrate crystal as inputs. As the SAW velocity dispersion was calculated for different angular positions of the sample, the stiffness matrix of the substrate was adapted using the Bond method to account for the angle $\theta$.

For a series of velocities $v$ (which correspond to frequencies $\omega=v k$ where $k=2 \pi / \lambda)$, the following steps were taken. The roots of $\Delta \Gamma$-see Eq. (2) - were calculated to find the wavenumbers in the $z$-direction, $\xi_{j, s / i n / c}$. Subsequently, the vectors $U_{m j, s / i n / c}$-see Eq. (3)-were determined by applying singular value decomposition to $\Gamma$. It was then possible to calculate all components of $R$, the $\mathrm{BC}$ matrix, and retain $\Delta R$ for every velocity and angle. To extract the SAW velocity dispersion from this matrix $\Delta R(v, \theta)$, the local minima were examined, because it is impossible for $\Delta R$ to be exactly 0 in a numerical approach. The selection of the local minima was done for every angle $\left(\theta_{i}\right)$ separately. This was accomplished as follows: the velocity corresponding to the deepest minimum (i.e., the local minimum with the smallest value for $\left.\Delta R\left(v, \theta_{i}\right)\right)$ and the velocity corresponding to the minimum resulting in the highest normal displacement were retained. The first criterion corresponded to the best solution of $\Delta R=0$, while the second criterion was employed in Ref. 25 and was relevant for the analysis of ISTS data, where the signal amplitude depended on the normal displacement of the sample surface. If both selected velocities were the same, then this velocity was retained as a part of the SAW velocity dispersion. If these velocities were different, the velocity corresponding to the local minimum with the smallest in plane parallel displacement-i.e., $u_{y}(z=0)$-was selected.

Finally, a SAW velocity dispersion curve, $c_{\mathrm{SAW}}(\theta)$, was obtained for the chosen wavelength $\lambda$. Repeating this procedure for all $\lambda$ 's used in the experiments, a 2D map of the SAW velocity, $c_{\mathrm{SAW}}(\lambda, \theta)$, could be calculated.

\section{EXPERIMENTAL RESULTS}

In Sec. IV A, a brief explanation of the ISTS technique is presented. The experimental results and analysis are presented in Sec. IV B. The obtained values for $E$ and $\psi$ are confirmed by two control measurements (Sec. IV C).

\section{A. Experimental setup}

The experimental setup used in the ISTS technique is depicted in Figure 2 and will be briefly described here. For a more detailed description, the reader is referred to Ref. 26. A $10 \mathrm{ps}$ pulsed laser beam $(1047 \mathrm{~nm})$ was focused, by a cylindrical lens, into a horizontal line onto a surface relief square wave grating. The grating was optimized for diffraction of light with a $532 \mathrm{~nm}$ optical wavelength, hence roughly $80 \%$ of the incident light was sent to the +1 and -1 diffraction order. All beams, i.e., the zeroth order, first orders and higher orders (which carry only a small percentage of the light intensity), recombine at the sample surface to create an interference pattern with a specific fringe distance that depends on the diffraction grating and the optical magnification factor

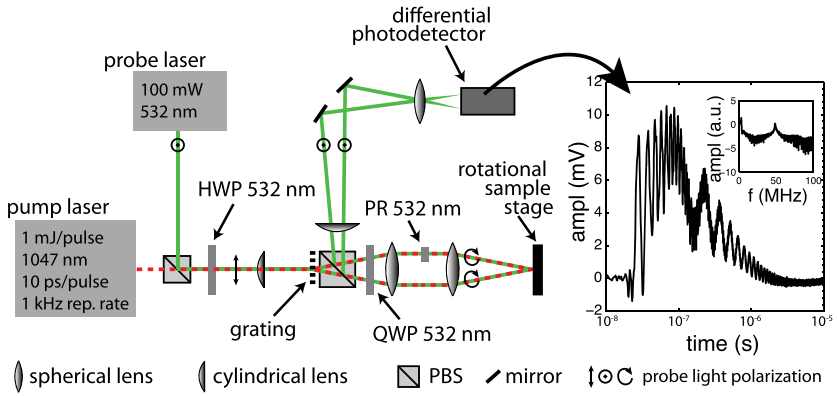

FIG. 2. ISTS setup. The incoming beams are diffracted into a 0th order, +1 , and -1 diffraction orders and higher orders. For the sake of clarity, only the +1 and -1 diffraction orders are presented here because both pump and probe laser beams are diffracted most efficiently to these orders. The signal on the right was obtained from SAWs with a grating wavelength of $100 \mu \mathrm{m}$.

of the two-lens telescope. At the sample surface, a part of the incident light was absorbed, resulting in impulsive heating. The accompanying sudden thermal expansion launches counter-propagating SAWs with a wavelength corresponding to the interference fringe distance. This results in a standing wave pattern that oscillates at a central frequency, corresponding to the ratio of the dispersive phase velocity and the excited wavelength.

The standing acoustic waves and the thermal diffusion driven washing-out of the induced thermal pattern were monitored by a cw probe laser. The probe laser beam followed the same optical path as the pump laser beam and thus was also focussed into an interference pattern at the excitation location. At the sample surface, the standing SAW pattern acted as a temporally modulated diffraction grating for the two probe beams, which are thus partially diffracted and partially reflected. Recombining the two pairs of reflected and diffracted beams resulted in heterodyne detection $^{27}$ of the standing wave pattern. The intensity modulations were recorded by two Si pin-photodiodes (Hamamatsu S5973) and a high speed GHz amplifier (Femto series HSA). The two signals were $180^{\circ}$ out of phase due to the introduction of a phase retarder (PR) in the path of one of the probe beams. Subtracting the two heterodyne signals resulted in a doubling of the signal amplitude.

The sample was mounted on a rotation stage, which allowed for the creation and detection of SAWs in any direction on the sample surface. By recording the time signal on an oscilloscope, the central frequency could be obtained after a fast Fourier transform (FFT) analysis. Combined with the experimentally known wavelength of the SAW, the phase velocity could be determined for all excited wavelengths and for any direction.

In Figure 2, a time signal is shown. Note that there are two frequency components present. The high frequency oscillation is the SAW of interest here, while the low frequency oscillation is the Scholte wave (a wave that travels at the interface of the solid sample and the air, at the speed of sound in $\operatorname{air}^{28}$ ). The high frequency oscillation results in the peak around $50 \mathrm{MHz}$ in the FFT spectrum, and the low frequency oscillation results in a peak around $3.4 \mathrm{MHz}$. As the imposed grating wavelength was $100 \mu \mathrm{m}$, this results in a SAW velocity of roughly $5000 \mathrm{~m} \cdot \mathrm{s}^{-1}$ and a Scholte wave 
velocity of roughly $340 \mathrm{~m} \cdot \mathrm{s}^{-1}$. This value is the speed of sound in air at room temperature, thus confirming the value of the imposed grating wavelength.

\section{B. SAW velocity dispersion results and analysis}

The obtained experimental data are shown in Figure 3, where the SAW velocity dependence on both the direction and $\lambda$ of the imposed grating is visible. The $90^{\circ}$ symmetry of the $\mathrm{Si}(001)$ substrate is visible in all imposed wavelengths. This is because at wavelengths much smaller than the coating thickness, the angular dispersion would disappear as the SAW velocity becomes the same for all directions. This velocity would be equal to the Rayleigh velocity of the isotropically assumed $\mathrm{MnO}_{2}$.

The SAW velocity depends on the Young's modulus $E$, Poisson's ratio $\nu$, the density $\rho$, and the thickness $L$ of the coating, as well as on other parameters (e.g., the properties of the substrate and the intermediate layer). In order to obtain a reliable fit for all four parameters, the SAW velocity dispersion was calculated based on sets of these parameters $(E, \nu, \rho, L)$. These calculated SAW velocity dispersion curves were compared to the experimental data to obtain a least squares error (LSE)

$$
\chi_{\text {mnop }}^{2}=\frac{1}{N} \sum_{i=1}^{N}\left(c_{\mathrm{SAW}}^{\mathrm{calc}}\left(\lambda_{i}, \theta_{i}, E_{m}, \nu_{n}, \rho_{o}, L_{p}\right)-c_{\mathrm{SAW}}^{\exp }\left(\lambda_{i}, \theta_{i}\right)\right)^{2}
$$

where $\lambda_{i}$ are the wavelengths and $\theta_{i}$ are the angles with $\operatorname{Si}[100]$ that were probed in the experiment, $c_{\mathrm{SAW}}^{\exp }\left(\lambda_{i}, \theta_{i}\right)$ is the experimentally obtained SAW velocity dispersion, $E_{m}, \nu_{n}, \rho_{o}, L_{p}$ are the values used to calculate the SAW velocity dispersion curves, and $N$ is the number of points used in the fit. The $\chi_{\text {mnop }}^{2}$ value was calculated for all combinations of $\left(E_{m}, \nu_{n}, \rho_{o}, L_{p}\right)$, where $E_{m}$ was varied from $10 \mathrm{GPa}$

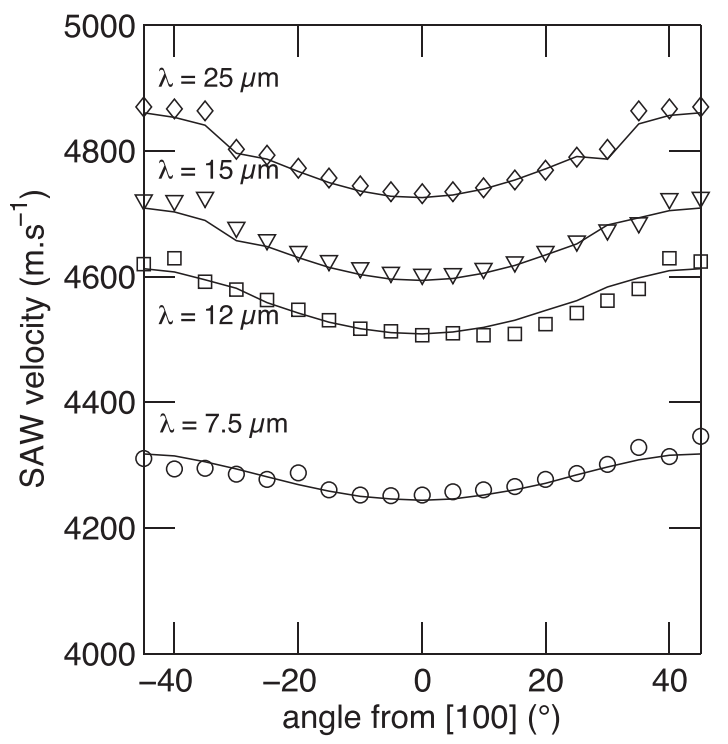

FIG. 3. Experimentally obtained SAW data represented by circles, squares, triangles, and diamonds for a wavelength of $\lambda=7.5 \mu \mathrm{m}, \lambda=12 \mu \mathrm{m}$, $\lambda=15 \mu \mathrm{m}$, and $\lambda=25 \mu \mathrm{m}$, respectively. The black lines depict the fitted dispersion. to $40 \mathrm{GPa}$ in 13 steps, $\nu_{n}$ was varied from 0.1 to 0.3 in 13 steps, $\rho_{o}$ was varied from $2200 \mathrm{~kg} \cdot \mathrm{m}^{-3}$ to $3500 \mathrm{~kg} \cdot \mathrm{m}^{-3}$ in 13 steps and $L_{p}$ was varied from $400 \mathrm{~nm}$ to $600 \mathrm{~nm}$ in 13 steps. Figure 4 shows a 2D representation of the $4 \mathrm{D}(13 \times 13 \times 13$ $\times 13)$ matrix containing the values of $\chi_{m n o p}^{2}$. Each pixel was assigned a color representing the magnitude of the $\chi^{2}$ value (black, red, yellow and white, corresponding to increasingly larger $\chi^{2}$ values). The set of parameters $\left(E^{*}, \nu^{*}, \rho^{*}, L^{*}\right)$ is the one that results in the smallest $\chi^{2}$ value (i.e., which results in the best fit of the experimental data).

From this 4D matrix, least squares (LS) and most squares (MS) curves ${ }^{16}$ can be constructed for all parameters (Figure 5). The LS curves were constructed by varying one parameter (e.g., $E$ ) and keeping all other parameters at the values that resulted in the best fit, $\left(\nu^{*}, \rho^{*}, L^{*}\right)$. The values of the $\chi^{2}$ matrix that correspond to these points were used to construct $\chi_{\mathrm{LS}}^{2}(p)$, where $p$ indicates the parameter that was varied. This can be done for all parameters and resulted in parabolas that be described by ${ }^{29}$

$$
\frac{\chi_{\mathrm{LS}}^{2}(p)}{\chi^{2}\left(p^{*}\right)}=A\left(p-p^{*}\right)^{2}+1
$$

where $A \geq 0$ is a constant related to the fitting uncertainty and $p$ is the parameter for which the LS curve is constructed. From Eq. (10), it is obvious that the parabolic function has a minimum at $p^{*}$, which is the best fitting value for the parameter $p$.

The MS curves were constructed in a more elaborate way. Again, one parameter is varied, but for every change in that parameter, the point $\left(\nu_{p}^{*}, \rho_{p}^{*}, L_{p}^{*}\right)$ with the smallest $\chi^{2}$ value is sought. These $\chi^{2}$ values are used to construct $\chi_{\mathrm{MS}}^{2}(p)$, which is a parabolic function described by

$$
\frac{\chi_{\mathrm{MS}}^{2}(p)}{\chi^{2}\left(p^{*}\right)}=B\left(p-p^{*}\right)^{2}+1,
$$

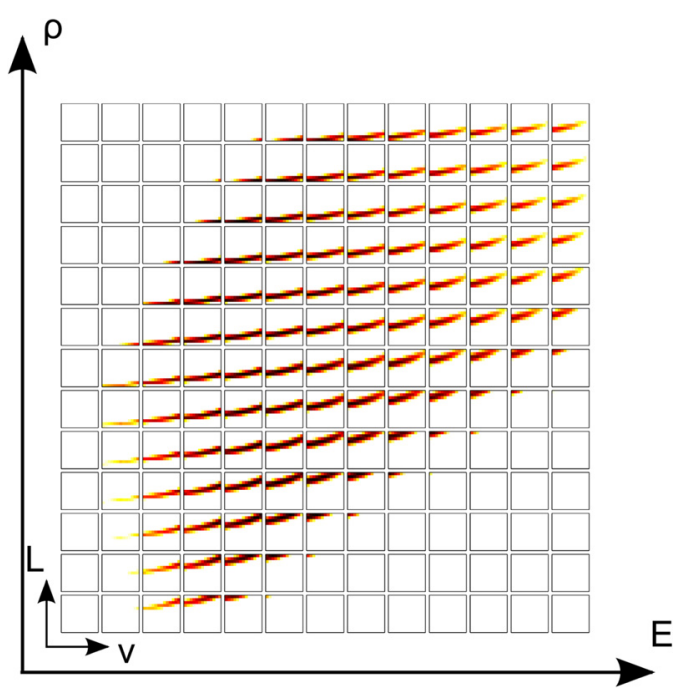

FIG. 4. 2D representation of a 4D matrix that contains values of $\chi^{2}$ between the experimentally observed SAW velocity dispersion and the simulated dispersion curves for different values of $(E, \nu, \rho, L)$. Each pixel in this $13 \times 13$ grid of $13 \times 13$ pixel images was assigned a color. Black, red, yellow, and white correspond to increasingly larger $\chi^{2}$ values. The small figure squares represent the dependence of the $\chi^{2}$ magnitude on $L$ and $\nu$, for an $E$ and $\rho$ value on the respective grid position. 
where $B \geq 0$ is a constant related to combination of the fitting uncertainty and the interdependence of that parameter with the other parameters. In other words, if the inverse problem is uniquely defined, i.e., if there exists only one set of $\left(E^{*}, \nu^{*}, \rho^{*}, L^{*}\right)$ that minimizes the $\chi^{2}$ cost function, a parabolic minimum in the most squares curve exists-i.e., $B>0$-around the best fitting value for all four parameters. Inversely, if the effect of changing one parameter (e.g., $E$ ) can be counteracted by changing the other parameters $(\nu, \rho$, and $L$ ), then the most squares curve becomes flat (and thus $B=0$ ), so that no value can be retrieved for that parameter and the error estimate becomes infinite.

It can be shown ${ }^{29}$ that the least and most squares uncertainties on the obtained fit values are

$$
\begin{aligned}
\sigma_{p, \mathrm{LS}} & =\frac{1}{\sqrt{N-P}} \frac{1}{\sqrt{A}}, \\
\sigma_{p, \mathrm{MS}} & =\frac{1}{\sqrt{N-P}} \frac{1}{\sqrt{B}},
\end{aligned}
$$

where $P$ is the number of parameters used in the fitting process. The values of $A^{-\frac{1}{2}}$ and $B^{-\frac{1}{2}}$ were the respective points $\left(p-p^{*}\right)$ where the LS and MS parabolas have a value of 2 .

The curves shown in Figure 5 reveal that the MS curves for $\nu$ and $L$ are flat, and that, it is therefore, not possible to perform a reliable fit for these two parameters. Consequently, a more detailed $\chi^{2}$ LSE map was calculated where only $E$ and $\rho$ are varied while the Poisson's ratio was ad hoc assumed to be $\nu=0.2$ and the thickness was known from the SEM image to be $L=500 \mathrm{~nm}$. The resulting map is shown in Figure 6, where an absolute minimum is visible. On the right and bottom of the map, the extracted LS and MS curves are shown, from which the values of $E$ and $\rho$ and the error estimates on these values were extracted (see
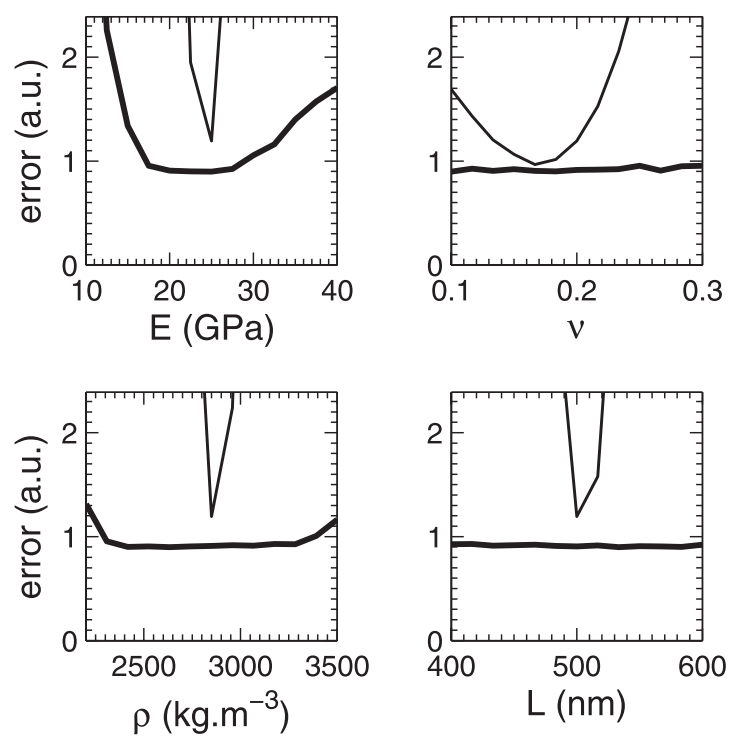

FIG. 5. Least and most squares curves (thin and thick lines, respectively) for all parameters that describe the elastic behavior of the $\mathrm{MnO}_{2}$ coating. The poor resolution is due to the limited number of points in the $13 \times 13$ $\times 13 \times 13$ LSE matrix represented in Figure 4 . The LS and MS curves of $E$ and $\rho$ are calculated in more detail in Figure 6.

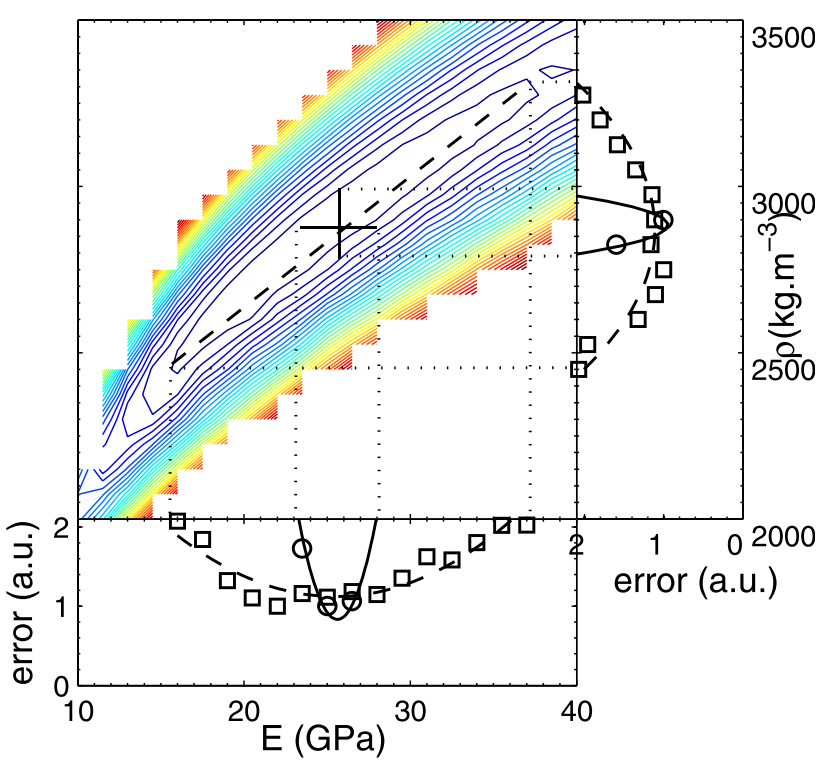

FIG. 6. $\chi^{2}$ landscape between the experimentally observed SAW dispersion and the simulated dispersion curves as a function of $E$ and $\rho$. The contour lines show that a true minimum is present at $E=25 \mathrm{GPa}$ and $\rho=2900 \mathrm{~kg} \cdot \mathrm{m}^{-3}$. The ragged edges are due to the limited number of points $(21 \times 21$ pixels $)$ used in the calculation of this figure. At the bottom and right of the LSE map, the extracted least and most square curves are presented. The squares and circles are, respectively, the $\chi_{\mathrm{LS}}^{2}$ and $\chi_{\mathrm{MS}}^{2}$ values that are extracted from the LSE landscape. The solid and dashed lines are the quadratic fits using Eqs. (10) and (11). The solid lines are calculated using more points than are visible.

Table II). From the density, the porosity of the film was calculated as $\psi=1-\frac{\rho}{\rho_{0}}$, where $\rho_{0}=5030 \mathrm{~kg} \cdot \mathrm{m}^{-3}$ is the density of bulk $\mathrm{MnO}_{2}$.

\section{Validation through independent methods}

The value of $E$ was independently determined through nano indentation to be $E=(26 \pm 3.5) \mathrm{GPa}$. The porosity was independently determined through a comparison of the measured thickness, using the SEM image of the crosssection and the quantity of $\mathrm{MnO}_{2}$ obtained by Rutherford Back Scattering (RBS). The quantity of $\mathrm{MnO}_{2}$, as determined by RBS, was equivalent to a thickness of $(251 \pm 1)$ $\mathrm{nm}$, assuming a $0 \%$ porosity film, i.e., with the density of nonporous bulk $\mathrm{MnO}_{2}\left(\rho_{0}=5030 \mathrm{~kg} \cdot \mathrm{m}^{-3}\right)$. From the SEM images, a film thickness of $(460 \pm 55) \mathrm{nm}$ was obtained. Hence the porosity was determined to be $\psi=(45 \pm 6) \%$.

These values agree well with the values extracted from the SAW velocity dispersion fitting process as presented in this work.

TABLE II. Best fitting values of the Young's modulus and density. These were obtained under the assumption that $L=500 \mathrm{~nm}$ and $\nu=0.2$. The control values were obtained by independent techniques as is explained in Sec. IV C.

\begin{tabular}{lrllr}
\hline & Fit value & $\sigma_{\mathrm{LS}}$ & $\sigma_{\mathrm{MS}}$ & control \\
\hline$E(\mathrm{GPa})$ & 25 & \pm 0.2 & \pm 1 & $26 \pm 3.5$ \\
$\rho\left(\mathrm{kg} \cdot \mathrm{m}^{-3}\right)$ & 2900 & \pm 20 & \pm 40 & $2750 \pm 300$ \\
$\psi(\%)$ & 42 & \pm 0.5 & \pm 1 & $45 \pm 6$ \\
\hline \hline
\end{tabular}




\section{CONCLUSION AND DISCUSSION}

From the SAW velocity dispersion, obtained by ISTS, a value for the Young's modulus $(E=25 \pm 1 \mathrm{GPa})$ and the porosity $(\psi=42 \pm 1 \%)$ was extracted for a $\mathrm{MnO}_{2}$ coating. In the analysis, the wavelength dependence of the SAW velocity anisotropy, which was a consequence of the wavelength dependent SAW penetration depth and of the samples consisting of the isotropic coating material of interest on top of an anisotropic silicon substrate, was exploited to a maximum extent. The uncertainties on the fitting parameters were extracted from a rigorous most squares analysis. Additionally, the values were confirmed by two independent techniques, namely nano indentation and a comparison between the thickness obtained from SEM and RBS for the values of $E$ and $\psi$, respectively. This is, to the best of the authors knowledge, the first reported value of the elastic properties of a thin, porous $\mathrm{MnO}_{2}$ film. The results demonstrate that the presented approach is able to, simultaneously and non-destructively, estimate a value for the Young's modulus and the porosity, with uncertainties smaller than the ones of classical state of the art techniques.

\section{ACKNOWLEDGMENTS}

The authors would like to thank M. H. van der Veen, IMEC, for the SEM images, and Sebastiaan Creten for the making of Figure 2. This work was possible with the financial support from the KU Leuven research Project No. OT/11/064, FWO Project No. KAN2010 1.5.168.10.

${ }^{1}$ P. H. Notten, F. Roozeboom, R. A. Niessen, and L. Baggetto, Adv. Mater. 19, 4564 (2007).

${ }^{2}$ M. M. Thackeray, Prog. Solid State Chem. 25, 1 (1997).

${ }^{3}$ W. Wei, X. Cui, W. Chen, and D. G. Ivey, Chem. Soc. Rev. 40, 1697 (2011).

${ }^{4}$ S. Sarciaux, A. Le Gal La Salle, A. Verbaere, Y. Piffard, and D. Guyomard, J. Power Sour. 81-82, 656 (1999).

${ }^{5}$ S. Sarciaux, A. Le Gal La Salle, A. Verbaere, Y. Piffard, and D. Guyomard, J. Power Sour. 81-82, 661 (1999).
${ }^{6}$ J. Rogers, A. Maznev, M. Banet, and K. Nelson, Annu. Rev. Mater. Sci. 30, 117 (2000).

${ }^{7}$ T. Valier-Brasier, T. Dehoux, and B. Audoin, J. Appl. Phys. 112, 024904 (2012).

${ }^{8}$ R. Lee and R. M. White, Appl. Phys. Lett. 12, 12 (1968).

${ }^{9}$ K. Nelson, D. Lutz, M. Fayer, and L. Madison, Phys. Rev. B 24, 3261 (1981).

${ }^{10}$ B. Sun, J. M. Winey, N. Hemmi, Z. A. Dreger, K. A. Zimmerman, Y. M. Gupta, D. H. Torchinsky, and K. A. Nelson, J. Appl. Phys. 104, 073517 (2008).

${ }^{11}$ C. Glorieux, K. Nelson, G. Hinze, and M. Fayer, J. Chem. Phys. 116, 3384 (2002).

${ }^{12}$ Y. Yang, L. J. Muller, and K. A. Nelson, in "Materials Research Society Symposium Proceedings," edited by H. Z. Cummins, D. J. Durian, D. L. Johnson, and H. E. Stanley (Mater. Res. Soc. Symp. Proc., 1996), Vol. 407, pp. 145-154.

${ }^{13}$ D. Paolucci and K. Nelson, J. Chem. Phys. 112, 6725 (2000).

${ }^{14}$ J. Sermeus, O. Matsuda, R. Salenbien, B. Verstraeten, J. Fivez, and C. Glorieux, Int. J. Thermophys. 33, 2145 (2012).

${ }^{15}$ C. Glorieux, K. V. de Rostyne, J. Goossens, G. Shkerdin, W. Lauriks, and K. Nelson, J. Appl. Phys. 99, 013511 (2006).

${ }^{16}$ R. Salenbien, R. Cote, J. Goossens, P. Limaye, R. Labie, and C. Glorieux, J. Appl. Phys. 109, 093104 (2011).

${ }^{17}$ J. Rogers and K. Nelson, J. Appl. Phys. 75, 1534 (1994).

${ }^{18}$ J. Deboucq, M. Duquennoy, M. Ouaftouh, F. Jenot, J. Carlier, and M. Ourak, Rev. Sci. Instrum. 82, 064905 (2011).

${ }^{19}$ D. Cott, M. Verheijen, O. Richard, I. Radu, S. De Gendt, S. V. Elshocht, and P. Vereecken, Carbon 58, 59 (2013).

${ }^{20}$ M. A. Hopcroft, W. D. Nix, and T. W. Kenny, J. Microelectromech. Syst. 19, 229 (2010).

${ }^{21}$ Z. Peng, H. Miao, L. Qi, J. Gong, S. Yang, and C. Liu, Chin. Sci. Bull. 48, 1316 (2003).

${ }^{22}$ B. Auld, Acoustic Fields and Waves in Solids (John Wiley \& Sons, 1973).

${ }^{23}$ W. L. Bond, Bell Syst. Tech. J. 22, 1 (1943).

${ }^{24}$ C. Glorieux, W. Gao, S. Kruger, K. V. de Rostyne, W. Lauriks, and J. Thoen, J. Appl. Phys. 88, 4394 (2000).

${ }^{25}$ W. Li, S. Sharples, R. Smith, M. Clark, and M. Somekh, J. Acoust. Soc. Am. 132, 738 (2012).

${ }^{26}$ B. Verstraeten, J. Sermeus, R. Salenbien, J. Fivez, G. Shkerdin, and C. Glorieux, "Determination of thermoelastic material properties by differential heterodyne detection of impulsive stimulated scattering," (to be published).

${ }^{27}$ A. Maznev, K. Nelson, and J. Rogers, Opt. Lett. 23, 1319 (1998).

${ }^{28}$ V. Gusev, C. Desmet, W. Lauriks, C. Glorieux, and J. Thoen, J. Acoust. Soc. Am. 100, 1514 (1996).

${ }^{29}$ P. R. Bevington and D. K. Robinson, Data Reduction and Error Analysis for the Physical Sciences (McGraw-Hill, New York, 1969), Vol. 336. 